\title{
Vortex dynamics in evolutive flows: A weakly chaotic phenomenon
}

\author{
Jacopo Bellazzini, ${ }^{1}$ Giulia Menconi, ${ }^{2}$ Massimiliano Ignaccolo, ${ }^{3}$ Guido Buresti, ${ }^{1}$ and Paolo Grigolini ${ }^{3,4,5}$ \\ ${ }^{1}$ Dipartimento di Ingegneria Aerospaziale, Università di Pisa, Via G. Caruso, 56100 Pisa, Italy \\ ${ }^{2}$ Dipartimento di Matematica Applicata, Università di Pisa, Via Bonanno Pisano 25/b, 56126 Pisa, Italy \\ ${ }^{3}$ Center for Nonlinear Science, University of North Texas, P.O. Box 311427, Denton, Texas 76203-1427, USA \\ ${ }^{4}$ Dipartimento di Fisica, Università di Pisa and INFM, Via Buonarroti 2, 56127 Pisa, Italy \\ ${ }^{5}$ Istituto dei Processi Chimico Fisici del Consiglio Nazionale delle Ricerche, Area della Ricerca di Pisa, Via Moruzzi 1, San Cataldo, \\ 56124 Pisa, Italy
}

(Received 16 December 2002; published 25 August 2003)

\begin{abstract}
We make use of a wavelet method to extract, from experimental velocity signals obtained in an evolutive flow, the dominating velocity components generated by vortex dynamics. We characterize the resulting time series complexity by means of a joint use of data compression and of an entropy diffusion method. We assess that the time series emerging from the wavelet analysis of the vortex dynamics is a weakly chaotic process with a vanishing Kolmogorov-Sinai entropy and a power-law growth of the information content. To reproduce the Fourier spectrum of the experimental signal, we adopt a harmonic dependence on time with a fluctuating frequency, ruled by an inverse power-law distribution of random events. The complexity of these fluctuations is determined by studying the corresponding artificial sequences. We reproduce satisfactorily both spectral and complex properties of the experimental signal by locating the complexity of the fluctuating process at the border between the stationary and the nonstationary states.
\end{abstract}

DOI: 10.1103/PhysRevE.68.026126

PACS number(s): 89.75.Fb, 05.45.-a, 47.27.-i

\section{INTRODUCTION}

In the past few years an increasing interest has been shown in studying physical processes with intermediate properties between chaotic and regular dynamics. Both theory and experimental measurements have been affording evidence for the existence of physical systems departing from the chaotic condition established by a positive Kolmogorov-Sinai (KS) entropy [1]. These systems, filling the gap between randomness and order, are characterized by vanishing KS entropy, and consequently, by a zero Lyapunov coefficient, but also by nonregular features. Indeed, a vanishing KS entropy does not necessarily imply a condition of order. Hereafter, we shall refer to these systems as weakly chaotic.

Nevertheless, we have to point out that this is still a somewhat vague definition, which applies to different physical conditions ranging from low-dimensional maps, with regular and random regions coexisting in the same phase space $[2,3]$, to systems yielding self-organization [4,5], which is a property incompatible with the condition of total randomness. For instance, in the case of low-dimensional systems, the condition of vanishing KS entropy applies to the classes of both anomalous kinetics along filamented surfaces [6] and of intermittent Manneville-like maps $[7,8]$.

Therefore, since the class of physical systems with vanishing KS entropy is huge, we need an even finer indicator to discriminate between different kinds of zero KS entropy dynamics. We choose the computable information content (CIC) (also known as the computable quantity related to the Kolmogorov complexity) as the indicator of the degree of complexity for weakly chaotic dynamical systems $[9,10]$. CIC measures the information that is necessary to describe $n$ steps of a typical orbit. Indeed, if we consider two dynamical systems with vanishing KS entropy, such as the Manneville map in the region $z \geqslant 2$ and the logistic map at the Feigenbaum point, the information grows in a different way. In the case of the Manneville map, described by $x_{n+1}=x_{n}$ $+x_{n}^{z}(\bmod 1)$, with $x \in[0,1]$, the information content grows as a power law $n^{\alpha}$ [where the exponent $\alpha$ is $\left.1 /(z-1)\right]$, as shown in Refs. $[8,11,12]$, while in the case of the logistic map at the chaos threshold, it grows as the logarithm of $n$ (see Sec. IV and Refs. [13,10,14]).

The aim of this paper is to study a fluid dynamical process in the outer shear layer of a coaxial jet flow: to be precise, to study the roll-up and pairing of vortices from an entropic point of view. We analyze experimental data from the jet flow to characterize the degree of complexity of the fluid dynamical process. We shall apply a wavelet-Hilbert analysis [15] on the experimental velocity signals, in order to extract and characterize only the fluctuations related to the two fluid dynamical processes (roll-up and pairing). The subsequent analysis of the reconstructed signals shows unambiguously a vanishing KS entropy. Furthermore, we prove that this condition of vanishing KS entropy is accompanied by a powerlaw growth of the computable information content.

Moreover, we propose a model displaying the same kind of complexity of the experimental data by means of a stochastic intermittent process ruled by an inverse power-law distribution of random events $\Psi(t) \sim t^{-\mu}$. This is the same waiting time distribution as that appearing in the Manneville map $[8,16]$ and in the class of random walks introduced in Ref. [17], which was proved to generate enhanced anomalous diffusion. This form of anomalous diffusion, when 2 $<\mu<3$, was called Lévy walk $[18,19]$ and used later as the prototype of the dynamical approach to Lévy diffusion (see, for instance, Refs. [20,21]). This type of dynamical processes led the authors of Ref. [22] to coin the term strange kinetics to point out the anomalous nature of the diffusion 


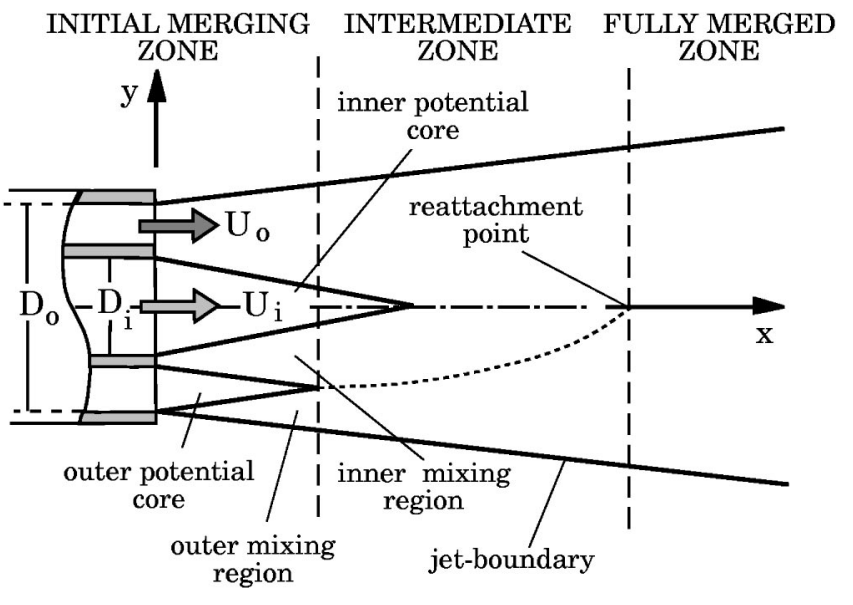

FIG. 1. Illustration of the coaxial jet.

process emerging from the waiting time distribution with divergent second moment.

To achieve our goal, we use three distinct techniques. The first one is the aforementioned wavelet-Hilbert analysis [15]. The second one is the diffusion entropy (DE) method [23,24] and, finally, the third one is the CIC method of Refs. $[9,10]$. This is the second example of a joint application of the DE and CIC methods. The first is given by the work of Ref. [24], where the reader can find a detailed discussion about the benefit stemming from the joint use of these two methods.

As for the experimental apparatus used to acquire the data to study the velocity signals are obtained in a coaxial jet flow (see the scheme in Fig. 1), described in more detail by Buresti et al. [25]. The analyzed configuration has an inner to outer jet diameter ratio $D_{o} / D_{i}=2.0$ (with $D_{i}=50 \mathrm{~mm}$ ), a velocity ratio $U_{o} / U_{i}=1.5$ (with $U_{o}=6 \mathrm{~m} / \mathrm{s}$ ), and wall thickness $L=5 \mathrm{~mm}$. The axial and radial velocity components, $u$ and $v$, were measured by means of $X$ hot wires placed inside the inner and the outer shear layers, at several axial positions downstream the jet outlet. The measurements at each point were carried out at least three times at a $5000 \mathrm{~Hz}$ sampling frequency for $20 \mathrm{~s}$.

The outline of the paper is as follows. To make it as self-contained as possible, in Secs. II-IV, we make a short review of the wavelet-Hilbert analysis, of the DE and of the CIC methods, respectively. In Sec. V, we illustrate the results of these techniques applied to the vortex dynamics under study in this paper. Finally, in Sec. VI, we discuss the results that were obtained.

\section{THE WAVELET-HILBERT ANALYSIS}

The experimental velocity signals acquired in many flow fields are characterized by fluctuations induced by the passage or by the oscillation of vortical structures. For instance, in the developing shear layers of a coaxial jet, the velocity signals are of increasing complexity with increasing distance from the jet outlet, and multiple dominating frequencies may be present, corresponding to fluctuations with different degrees of modulation both in amplitude and in frequency. These fluctuations are connected with the passage and dy- namics of vortical structures produced by the instability of the shear layers. Therefore, signal analysis procedures based only on conventional Fourier methods become largely inappropriate and different techniques, providing the time variation of the frequency and amplitude of the components present in the signals, should be used. A classical demodulation technique, on which the analysis procedures used in the present investigation are founded, shall now be briefly described. Any time-varying real signal $x(t)$ may be represented in the form

$$
x(t)=A(t) \cos [\varphi(t)] .
$$

This representation is not unique, but a so-called canonical pair may be defined for the quantities $(A, \varphi)$ if we introduce the associated complex analytic signal $Z_{x}(t)$ defined by

$$
Z_{x}(t)=A_{x}(t) e^{i \varphi_{x}(t)}=x(t)+i x_{H}(t),
$$

where $x_{H}(t)$ is the Hilbert transform of $x(t)$, given by

$$
x_{H}(t)=H[x(t)]=\frac{1}{\pi} P \int_{-\infty}^{+\infty} \frac{x(\tau)}{t-\tau} d \tau .
$$

The canonical representation of $x(t)$ is then

$$
x(t)=A_{x}(t) \cos \left[\varphi_{x}(t)\right],
$$

hereby yielding the following definition of instantaneous frequency of $x(t)$ :

$$
\nu_{x}(t)=\frac{1}{2 \pi} \frac{d \varphi_{x}(t)}{d t}
$$

This definition, which is perfectly correct from a mathematical point of view, acquires also a significant physical meaning when the analyzed signal is asymptotic, i.e., a sinusoidal signal which is slowly modulated in amplitude and frequency, $x(t)=A(t) \cos \left[\omega(t) t+\varphi_{0}\right]$. In this case, provided the modulation frequencies are sufficiently lower than the fundamental frequency of the signal, the time variation of amplitude and frequency may be directly recovered from the time variation of the modulus and of the phase derivative of the associated analytic signal.

However, this procedure is applicable only if the signal is composed of a single asymptotic component; conversely, if multiple components are present, other techniques must be devised. One of these [26] is based on the application of the continuous wavelet transform and exploits the strict analogy existing between the wavelet transform of a signal and the extraction of its associated analytic signal when the used wavelet is a complex analytic function (i.e., whose Fourier transform is zero for negative frequencies). Indeed, the single modulated components present in the signal may be extracted (provided they are asymptotic and sufficiently separated in frequency) from the restrictions of the wavelet transform to the curves where the wavelet modulus is maximum, which are called the ridges of the transform. More explicitly, if the signal is of the form 


$$
\begin{aligned}
x(t)=\sum_{n=1}^{N} x_{n}(t) & =\sum_{n=1}^{N} A_{n}(t) \cos \left[\varphi_{n}(t)\right] \\
& =\sum_{n=1}^{N} A_{n}(t) \cos \left[\omega_{n}(t) t+\varphi_{0 n}\right]
\end{aligned}
$$

and a Morlet wavelet $\psi(t)=e^{i \omega_{0} t} e^{-t^{2} / 2}$ is used, then the restrictions of the wavelet transform to the $N$ ridge curves $a$ $=a_{r n}(t)$ (where the wavelet scale $a$ is proportional to the inverse of the frequency) may be expressed as

$$
W_{x n}\left(a_{r n}(t), t\right)=\operatorname{Corr}(t) Z_{x n}+r(t),
$$

where the term $\operatorname{Corr}(t)$ is completely defined by the used wavelet and by the ridge values, and the residual $r(t)$ is negligible if the component is asymptotic. It is then possible to approximately obtain the analytic signals associated with the single components and to estimate the relevant frequency and amplitude modulation laws. However, some limitations exist in the frequency modulation laws that may be detected using this procedure [26]. In particular, we note that the signals that do not fit satisfactorily, the asymptotic condition make the wavelet ridge analysis much less accurate than the usual Hilbert demodulation technique, see Ref. [15]. Consequently, a new procedure was devised, which in a sense exploits the qualities of both techniques and which shall now be briefly described. The signal is first transformed using a Morlet wavelet and the ridges are approximately derived from the modulus maxima curves; in this procedure, a high value of the central frequency of the wavelet $\omega_{0}$ is used, in order to assure a high frequency resolution and the reduction of the interference effects between adjacent components. The wavelet maps are then filtered, neglecting the coefficients outside a band around the dominating ridge, and an inverse transform is applied. The extracted signal may then be subtracted from the original one and the procedure may be repeated until all the detectable components are extracted. The Hilbert transform technique is then applied to each component, in order to obtain the required modulation laws. In the latter step, further filtering may be used, considering only the time intervals in which the modulus of the associated analytic signal is higher than a given threshold (in terms of a given percent of its mean value). This avoids spurious large fluctuations of the instantaneous frequency and the final statistical analysis may be restricted to those intervals of time in which this frequency is physically meaningful, in the sense that it may be confidently associated with fluctuations produced by a fluid dynamical mechanism, such as the passage or oscillation of vorticity structures. Obviously, in order to characterize their level of chaoticity, the single components obtained through the wavelet filtering procedure may also be analyzed with different techniques, as, for instance, those described in the following sections.

\section{DIFFUSION ENTROPY METHOD}

This technique of analysis of time series was introduced originally in the paper of Ref. [23], whose authors coined, in fact, the term diffusion entropy. It became clear immediately afterwards [27] that the DE method is an efficient method of establishing the scaling value if the scaling condition applies, regardless of the scaling origin, and even when the scaling condition, according to the generalized central limit theorem (GCLT) [28], yields a divergent variance. Given a discrete signal $\left\{x_{i}\right\}_{i=1, N}$, we interpret it as a set of diffusion generating fluctuations. The collection of these fluctuations yield a single diffusion trajectory. We can create a set of many different diffusion trajectories by means of moving windows of size $t$ with $1<t<N$. We generate $N-t+1$ trajectories considering the sum

$$
y_{j}(t)=\sum_{i=j}^{i=j+t} x_{i}
$$

Each diffusive trajectory can be thought of as the final position of a walker which jumped for $t$ time steps. Let $p(y, t)$ be the probability to be at position $y$ after $t$ time steps. If the scaling condition applies to the asymptotic time limit, $p(y, t)$ is expected to fulfill the following condition:

$$
p(y, t)=\frac{1}{t^{\delta}} F\left(\frac{y}{t^{\delta}}\right) .
$$

Some of the processes of anomalous diffusion fit the prediction of the GCLT, thereby assigning to the function $F(y)$ the structure of a Lévy distribution with a diverging second moment. This is a problem for the techniques of analysis based on the observation of the second moment. It is not a problem for the DE, which evaluates the scaling parameter $\delta$ from the Shannon entropy of the probability $p(y, t)$ :

$$
S(t)=-\int_{-\infty}^{+\infty} p(y, t) \ln [p(y, t)] d z .
$$

Indeed, it is straightforward to prove that when the scaling condition of Eq. (3) holds, Eq. (4) yields $S(t)=A+\delta \ln t$, where $A$ is the Shannon entropy of the generating function $F$. Thus, the scaling parameter $\delta$ is easily evaluated by plotting the Shannon entropy in a diagram with linear ordinate and a logarithmic abscissa. The Shannon entropy, as a function of time, becomes a straight line whose slope is the scaling parameter $\delta$.

It is important to point out that in the case where the time series under study is a periodic process, the diffusion entropy, starting from a small initial value, reaches a maximum, then it regresses to the initial value, at a time corresponding to the period of the process under study [24]. Then, moving from this value, the diffusion entropy increases again up to a maximum which has the same value as the earlier maximum, and so on. In the case of the logistic map at the chaos threshold, the diffusion entropy exhibits a behavior very similar to that of a periodic process. The maxima still lie on a horizontal line, but the regressions to the minima are not complete as in the periodic case and occur in a disordered way [24].

In the case of periodic processes, the initial entropy increase is due to the fact that the moving window technique, 
adopted to create a large number of different diffusion trajectories, is equivalent to setting uncertainty on the initial condition.

\section{COMPUTABLE INFORMATION CONTENT METHOD}

The second method of analysis used in this paper aims at establishing a direct contact with algorithmic information content (also known as Kolmogorov complexity). In this second method, the basic notion is the notion of information. Given a finite string $s$ (namely, a finite sequence of symbols taken in a given alphabet), the intuitive meaning of quantity of information $I_{\mathrm{AIC}}(s)$ contained in $s$ is the length of the shortest binary message from which we can reconstruct $s$ (e.g., if we are working on a computer that binary message is a program $p$ that outputs $s$ ). This concept is expressed by the notion of algorithmic information content (AIC). We limit ourselves to illustrating the intuitive definition of the concept of AIC; for further details see Refs. $[29,10]$ and related references.

By definition, the shortest program $p$ which outputs the string $s$ is a sort of optimal encoding of $s$ : the information that is necessary to reconstruct the string is contained in the program. Unfortunately, this coding procedure cannot be performed on a generic string by any algorithm: the algorithmic information content is a quantity which is not computable by any algorithm (see Chaitin theorem in Ref. [29]).

Another measure of the information content of a finite string can also be defined by a loss-less data compression algorithm $Z$ satisfying some suitable optimality properties which we shall not specify here. Details are discussed in Ref. [10]. We can define the information content of the string $s$ as the binary length of the compressed string $Z(s)$, namely,

$$
I_{Z}(s)=|Z(s)| \text {. }
$$

The advantage of using a compression algorithm lies in the fact that, this way, the information content $I_{Z}(s)$ turns out to be a computable function. For this reason, we shall call it computable information content.

The notion of information is strongly related to chaos, unpredictability and instability of the behavior of dynamical systems. The KS entropy can be interpreted as the average measure of information that is necessary to describe a step of the evolution of a dynamical system.

We have seen that the information content of a string can be defined either with probabilistic methods (following the Shannon theory) or using the AIC or the CIC. Similarly, also the KS entropy of a dynamical system can be defined in different ways. The probabilistic method is the usual one, the AIC method has been introduced by Brudno [30]; the CIC method has been introduced in Refs. [31,9]. So, in principle, it is possible to define the entropy of a single trajectory of a dynamical system. There are different ways to do this (see Refs. [30,32,8,11,12]). In this paper, we make use of a method which can be implemented in numerical simulations. Now we shall describe it briefly.

Through the usual procedure of symbolic dynamics, given a discrete infinite trajectory $\bar{x}=\left\{x_{i}=T^{i}\left(x_{0}\right)\right\}_{i \geqslant 0}$ drawn from the dynamical system $(X, \mu, T)$, we consider a finite partition
$\mathcal{P}=\left(R_{1}, \ldots, R_{l}\right)$ of the dynamical system. In a standard way, we associate a string $\phi_{\mathcal{P}}(\bar{x})$ to the trajectory $\bar{x}$. We set $\phi_{\mathcal{P}}(\bar{x})=\left(s_{0}, s_{1}, \ldots, s_{k}, \ldots\right)$ if and only if

$$
\forall k \geqslant 0 \quad x_{k} \in R_{s_{k}}, \quad \text { where } s_{k} \in\{1, \ldots, l\} .
$$

We can define the information content $I(\bar{x}, \mathcal{P}, n)$ of the trajectory $\bar{x}$ with respect to the partition $\mathcal{P}$ in the following way:

$$
I(\bar{x}, \mathcal{P}, n):=\widetilde{I}\left(\phi_{\mathcal{P}}(\bar{x})^{n}\right),
$$

where $\phi_{\mathcal{P}}(\bar{x})^{n}$ is the string made of the first $n$ digits of the symbolic trajectory $\phi_{\mathcal{P}}(\bar{x})$. The information content $\widetilde{I}$ can be measured either via AIC or via CIC, so we have just defined $I_{\mathrm{AIC}}$ and $I_{Z}$ (respectively).

Let us assume that the compression algorithm $Z$ is optimal in the sense of Ref. [10]. We have the following results (see Ref. [10]).

Theorem 1. If $Z$ is an optimal coding, $(X, \mu, T)$ is an ergodic dynamical system and $\mathcal{P}$ is a measurable partition of $X$, then for $\mu$ almost all trajectories $\bar{x}$ drawn from the dynamical systems it holds

$$
I_{Z}(\bar{x}, \mathcal{P}, n)=I_{\mathrm{AIC}}(\bar{x}, \mathcal{P}, n)=n h_{\mu}(T, \mathcal{P})+o(n),
$$

where $h_{\mu}(T, \mathcal{P})$ is the Kolmogorov entropy of $(X, \mu, T)$ with respect to the measurable partition $\mathcal{P}$.

Theorem 2. Given the dynamical system $(X, T, \mu)$, if the measure $\mu$ on $X$ is $T$ invariant, then, if $Z$ is an optimal compression algorithm, for any measurable partition $\mathcal{P}$ it holds

$$
\begin{aligned}
& h_{\mu}(T, \mathcal{P})=\int_{X} \lim _{n \rightarrow+\infty} \sup _{n} \frac{I_{Z}(\bar{x}, \mathcal{P}, n)}{n} d \mu \\
& =\int_{X} \lim _{n \rightarrow+\infty} \sup \frac{I_{\mathrm{AIC}}(\bar{x}, \mathcal{P}, n)}{n} d \mu .
\end{aligned}
$$

Let us set $\lim \sup _{n \rightarrow+\infty} I(\bar{x}, \mathcal{P}, n) / n$ be the complexity $K(\bar{x}, \mathcal{P})$ of the trajectory $\bar{x}$ with respect to the partition $\mathcal{P}$. Theorem 2 shows that if a system has an invariant measure, its entropy with respect to a given partition can be found by averaging the complexity of its orbits over the invariant measure. Then, the entropy may be alternatively defined as the average orbit complexity. However, if we fix a single point, its orbit complexity is not yet well defined because it depends on the choice of a partition. We chose to get rid of this dependence by considering only a particular class of partitions and define the orbit complexity of a point as the supremum of the orbit complexity over that class.

Let $\beta_{i}$ be a family of measurable partitions such that $\lim _{i \rightarrow \infty} \operatorname{diam}\left(\beta_{i}\right)=0$. If we consider the quantity $\lim \sup _{i \rightarrow \infty} K_{Z}\left(\bar{x}, \beta_{i}\right)$, the following lemma holds (for the proof, see Ref. [10]).

Lemma 1. If $(X, \mu, T)$ is compact and ergodic, $Z$ is optimal, then for $\mu$ almost all points $x_{0} \in X$, for the trajectory $\bar{x}$ of starting point $x_{0}$ it holds 


$$
\lim _{i \rightarrow \infty} \sup K_{Z}\left(\bar{x}, \beta_{i}\right)=\lim _{i \rightarrow \infty} \sup K_{\mathrm{AIC}}\left(\bar{x}, \beta_{i}\right)=h_{\mu}(T) .
$$

Therefore, this lemma, under suitable conditions on the dynamical system, on the partitions and on the compression algorithm, permits to define almost everywhere the complexity of the orbit $\bar{x}$ of the starting point $x_{0}$ as $\lim \sup _{i \rightarrow \infty} K_{Z}\left(\bar{x}, \beta_{i}\right)=\lim \sup _{i \rightarrow \infty} K_{\mathrm{AIC}}\left(\bar{x}, \beta_{i}\right) . \quad$ These quantities asymptotically approach the KS entropy of the system. Indeed, the asymptotic behavior of $I(x, \mathcal{P}, n)$ gives an invariant of the dynamics which is finer than the KS entropy and is particularly relevant when the KS entropy is null.

It is well known that the KS entropy is related to the instability of the orbits. The exact relations between the KS entropy and the instability of the system are given by the Pesin theorem. We shall recall this theorem in the onedimensional case. Suppose that the average rate of separation of nearby starting orbits is exponential, namely,

$$
\Delta x(n) \simeq \Delta x(0) 2^{\lambda n} \quad \text { for } \Delta x(0) \ll 1,
$$

where $\Delta x(n)$ denotes the distance at time $n$ of two points initially at distance $\Delta x(0)$. The number $\lambda$ is called Lyapunov exponent; if $\lambda>0$ the system is unstable and $\lambda$ can be considered as a measure of its instability (or initial data sensitivity). The Pesin theorem implies that, under some regularity assumptions, $\lambda$ equals the KS entropy.

In weakly chaotic systems, the amount of information necessary to describe $n$ steps of a trajectory is less than linear in $n$ and the rate of separation of nearby starting orbits is less than exponential, then the KS entropy is not sensitive enough to distinguish the various examples of weakly chaotic dynamics since in every case the KS entropy is zero. Nevertheless, using the ideas we illustrated above, the relation between initial data sensitivity and information content of the orbits can be extended to these cases.

An example of such a generalization is given in Ref. [12]. In the following, for the sake of brevity we only briefly sketch the ideas underlying the results of Ref. [12], which are very deep and detailed. Let us consider a dynamical system $([0,1], T)$ where the transition map $T$ satisfies some constructivity properties (a constructive map is a map that can be defined using a finite amount of information) and the function $I(\bar{x}, \mathcal{P}, n)$ is defined using the AIC in a slightly different way than before (use open coverings instead of partitions). Provided the speed of separation of nearby starting orbits goes like $\Delta x(n) \simeq \Delta x(0) f(x, n)$, it has been proved in Ref. $[12]$ that under suitable assumptions on $f$, we have for almost all the points $x \in[0,1]$

$$
I(x, \mathcal{P}, n) \sim \ln [f(x, n)] .
$$

In the weakly chaotic case, the speed of separation of nearby starting orbits is less than exponential. In particular, if we have power-law sensitivity $\Delta x(n) \simeq \Delta x(0) n^{p}$, the information content of the trajectory is

$$
I(x, \mathcal{P}, n) \sim p \ln (n) .
$$

If we have a stretched exponential sensitivity $\Delta x(n)$ $\simeq \Delta x(0) 2^{\lambda n^{p}}, p<1$ (e.g., in the Manneville family with $z$ $>2$ ), the information content of the orbits shall increase with the power law:

$$
I(x, \mathcal{P}, n) \sim n^{p} .
$$

Since we have shown that the analysis of $I(\bar{x}, \mathcal{P}, n)$ gives useful information on the underlying dynamics and since $I(\bar{x}, \mathcal{P}, n)$ can be defined through the CIC methodology, it turns out that it can be used to analyze experimental data using a compression algorithm which is efficient enough and which is fast enough to analyze long strings of data. For the numerical experiments, we have used a compression algorithm called CASToRe, illustrated in an earlier publication [9]. The name of the algorithm is an acronym meaning compression algorithm, sensitive to regularity; the heuristic motivations for this name are explained in Ref. [10]. Therefore, in the following the algorithm $Z$ shall be the algorithm CASToRe.

\section{RESULTS}

As a significant earlier work on real fluid dynamical processes, we have in mind the pioneering work of Solomon, Weeks, and Swinney [33], who studied chaotic transport in a laminar fluid flow in a rotating annulus by tracking large numbers of tracer particles for long times. The remarkable result of that paper is the discovery that the particle motion can be easily described by means of a Lévy walk. In practice, these authors discovered strange kinetics. Our approach is slightly different. We do not adopt the Lagrangian approach to chaotic transport of Ref. [33], but rather the Euler perspective: we measure the velocity fluctuations in a suitable region of the flow.

In a jet flow, the shear layer between the so-called potential core and the ambient fluid is subjected to an instability, which produces a roll-up of the shear layer into vortical structures; moving downstream, these structures grow and normally undergo a process of pairing, through which two subsequent structures merge to form a larger one. Depending on the geometrical and fluid dynamical conditions, multiple pairings may occur before azimuthal instabilities lead to a complete mixing of the flow at the end of the jet core. The instability leading to roll-up is characterized by a dominating frequency, while the pairing process leads to a doubling of the period of passage of the structures, and then to the appearance of its subharmonics. In the case of a coaxial jet (Fig. 1), the situation is complicated by the presence of two cores and shear layers, which give rise to different families of vortices whose degree of interaction is a function of the ratios $D_{o} / D_{i}$ and $U_{o} / U_{i}$. In the present case $\left(D_{o} / D_{i}\right.$ $=2, U_{o} / U_{i}=1.5$ ), the stronger outer shear layer dominates the initial development of the flow and its dynamics is not far from that of a single jet [25]. In order to describe the performance of the different signal processing techniques in providing information on this dynamics, in the following we shall analyze the axial velocity signal $u$ obtained from the 


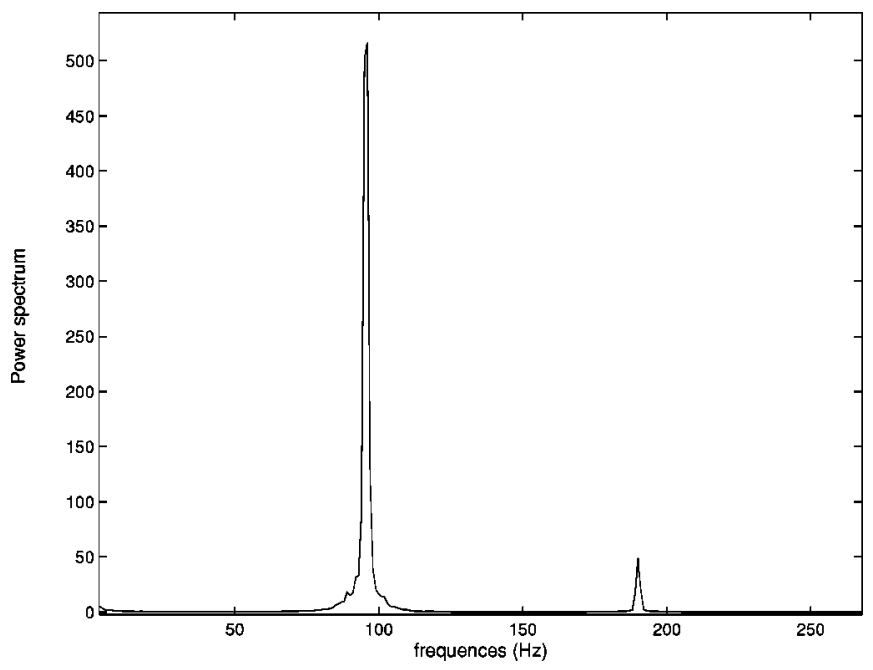

FIG. 2. Fourier power spectrum of the axial velocity $u$ at positions $x / D_{i}=1$ and $z / D_{i}=0.96$.

measurements in the position $z / D_{i}=1, y / D_{i}=0.96$, i.e., well inside the outer shear layer.

As shown in Fig. 2, the Fourier spectrum of this signal has two peaks, one at the fundamental frequency near 190 $\mathrm{Hz}$ (corresponding to a Strouhal number, based on $U_{o}$ and on the outer shear layer momentum thickness at the jet outlet, $\left.S t=f \theta / U_{o} \sim 0.0125\right)$ and a larger one at the subharmonic. This indicates that at this position the pairing process is already in an advanced stage, although not yet completed, as may be derived from the analysis of the radial velocity spectrum (not shown here for the sake of brevity), in which the peak at the fundamental frequency is still the dominating one.

The two components of the $u$ signal were extracted using the wavelet filtering procedure described above and we reconstructed the two signals $u_{a}$ and $u_{b}$, associated with the harmonic $\left(u_{a}, 190 \mathrm{~Hz}\right)$ and to the subharmonic $\left(u_{b}, 95 \mathrm{~Hz}\right)$ frequencies, respectively. The two reconstructed signals $u_{a}$ and $u_{b}$ were then analyzed using the different techniques described in the previous sections, starting from the Hilbert demodulation procedure.

Figure 3 shows a portion of the time variation of the in-

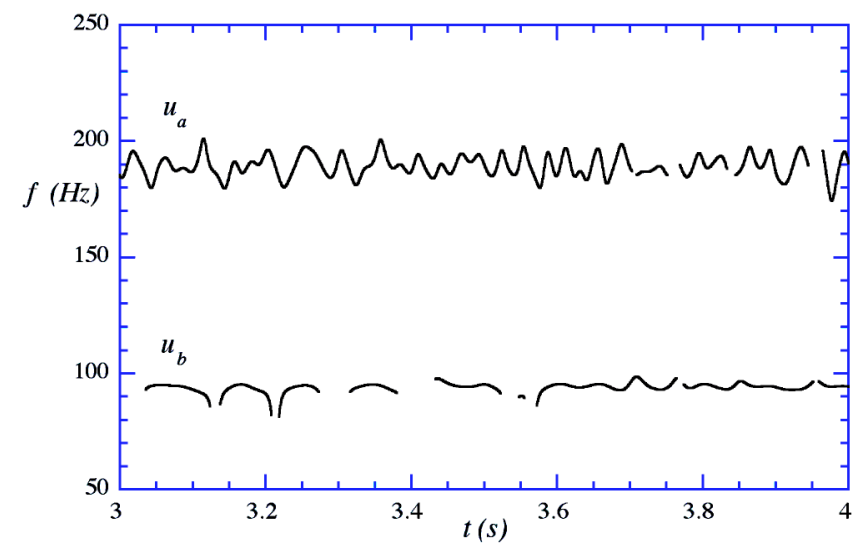

FIG. 3. Instantaneous frequencies of the components $u_{a}$ and $u_{b}$ as obtained using the wavelet-Hilbert procedure.

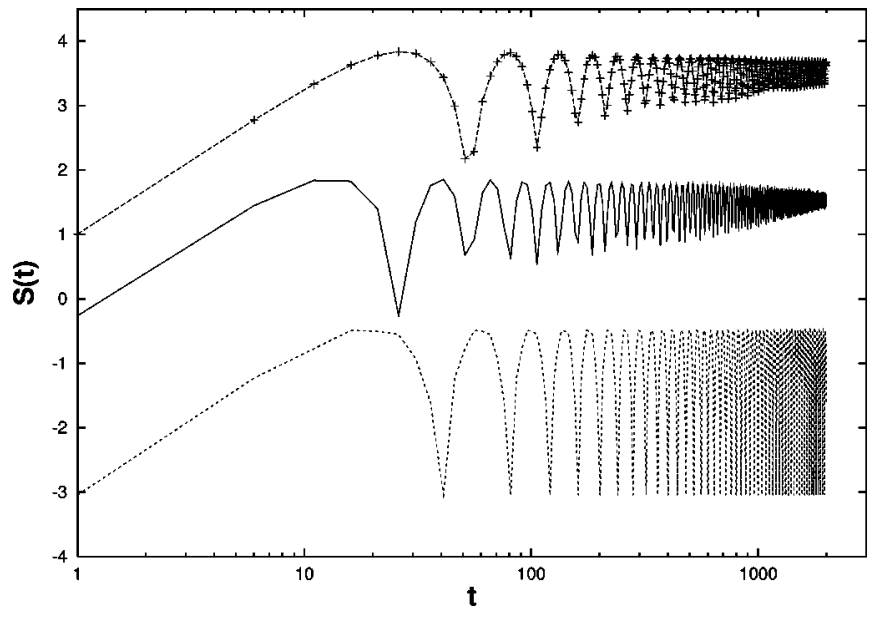

FIG. 4. Results of the DE analysis on $u_{b}$ (top graph, dashed line), $u_{a}$ (mid graph, solid line), and a purely periodic signal (bottom graph, dotted line). The scale is log linear.

stantaneous frequencies of the two components derived from their associated analytic signals. As already pointed out, to avoid unphysical variations of this frequency due to the degeneracy of the phase definition, the data are filtered considering only the time intervals in which the modulus of the analytic signal is above a given threshold (50\% of the modulus mean value in Fig. 3) and are then statistically analyzed. By this procedure, the mean values of the two frequencies were found to be 188.80 and $94.47 \mathrm{~Hz}$, with standard deviations of 4.8 and $0.93 \mathrm{~Hz}$, respectively. This technique also allows us to obtain the time variation of the ratio between the two instantaneous frequencies, whose mean value was found to be 2.002 , with a standard deviation of 0.05 , thus confirming that $u_{b}$ is the subharmonic of $u_{a}$. As can be seen from Fig. 3, the instantaneous frequencies vary in time with an irregular trend, and the same may be shown to be true for the modulus of the analytic signal, which corresponds to the amplitude of the original velocity component fluctuation. This feature is most probably due to the fact that the shape, size, radial position, and translation velocity of the vortical structures are not exactly constant in time. We are not able to find an explicit form of the irregular time evolution of instantaneous frequencies. However, the nature of the frequency fluctuations shall now be analyzed through the DE and CIC methods to shed light on the degree of complexity of the two fluid dynamical processes.

First, we have studied the two reconstructed signals $u_{a}$ and $u_{b}$ using the DE method. The results are illustrated in Fig. 4 and, to make our discussion more transparent, are compared to the result provided by the same method in the case of a purely periodic signal. The results of Fig. 4 deserve some detailed comments. First of all, we note that the two reconstructed signals yield a similar behavior, with an initial transient followed by an oscillatory behavior with the DE maxima lying on a horizontal line. This is a property shared by the periodic signal. It is evident, however, that the similarity with the periodic signal is not complete. Indeed, in the case of the periodic signal the regressions of the DE to the initial condition are complete, while in the case of the two 
reconstructed signals they are not; in fact, the regression intensities tend to decrease with time, suggesting that at infinite time the diffusion entropy might become rigorously constant. This perfect localization is similar to the slow occurrence of a collapse noticed years ago in the case of a problem of quantum chaos [34]. In that case, the dynamics under study was that of a $1 / 2$ dipole precessing about a magnetic field while undergoing the perturbation stemming from the harmonic motion of an oscillator. The entropy recursions to the initial condition are complete if the Larmor frequency is very small compared to oscillator frequency. In the case of resonance, however, chaos emerges and, consequently, incomplete regressions of decreasing intensity were recorded. All this suggests that the process under study is neither periodic nor quasiperiodic. In fact, in both cases the entropy regressions to the initial condition would be complete $[24,35]$.

In order to evaluate the degree of regularity of the two reconstructed signals, we have estimated, by means of the Rosenstein-Collins-De Luca algorithm [36], the dominant Lyapunov exponent of the two time series, which turned out to be zero for both sequences $u_{a}$ and $u_{b}$. Therefore, thanks to the Pesin theorem, we can say that the vortex dynamics in both cases of roll-up and pairing has null KS entropy. Together with the fact that the processes are not ordered, we may conclude that the vortex roll-up and pairing are weakly chaotic phenomena.

As a consequence, we classified the kind of complexity of the vortex dynamics by investigating the behavior of the information content of the reconstructed signals. We translated the time series into symbolic sequences by considering the interval $J_{a}=\left[\min \left\{u_{a}\right\}, \max \left\{u_{a}\right\}\right]$ (similarly, we defined $J_{b}$ ) and dividing it through several uniform partitions $\mathcal{P}_{k}$, with $k$ subintervals where $k \in\{2,4,8,16,32,64,100\}$. The different associated symbolic sequences have been compressed by means of the CASToRe algorithm. In all cases, the information content $I_{z}(n)$ as a function of the time steps $n$ has been explicitly calculated and was found to grow as a power law:

$$
I_{Z}(n) \sim C_{k} n^{\alpha} .
$$

The exponent $\alpha$ is 0.80 for $u_{a}$ and 0.83 for $u_{b}$; in both cases it does not depend on the particular partition used. The constant $C_{k}$ depends on the partition $\mathcal{P}_{k}$ and increases with the parameter $k$ (see Fig. 5). As illustrated in Sec. IV, the fact that the amount of information to describe $n$ steps of a trajectory is less than linear in $n$ is in complete agreement with the null KS entropy.

The discovery of the power-law growth of the information content, for both the time series $u_{a}$ and $u_{b}$, concludes our analysis on the experimental data. Now, we have to address the challenging task of building up a model reproducing the main properties of the vortex dynamics found so far. This model must explain the degree of complexity as it is indicated by the parameter $\alpha$ ranging from 0.80 to 0.83 , and at the same time it has to be compatible with the crucial property such as the peaked Fourier spectrum of Fig. 2. We propose a theoretical model driven by a hidden intermittent pro-

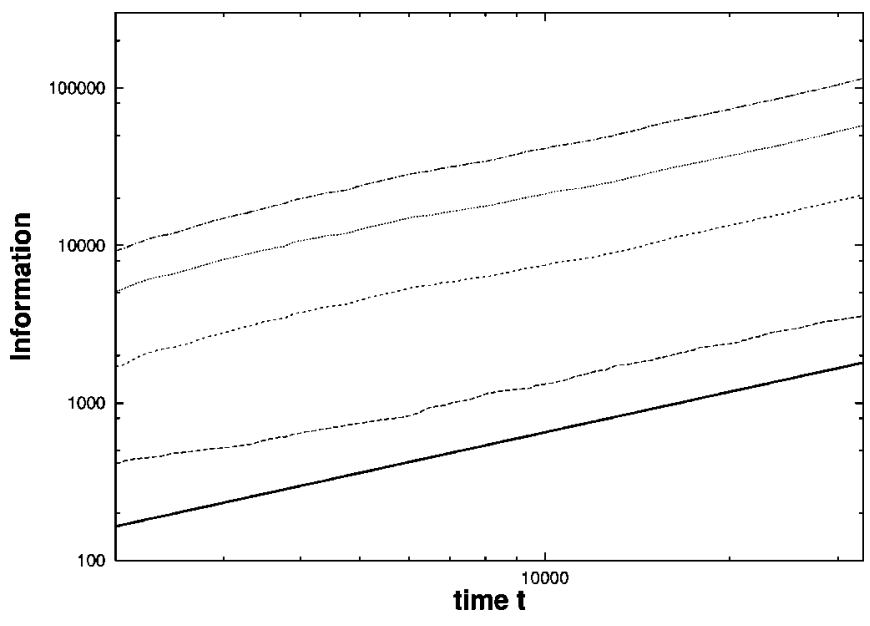

FIG. 5. The information content of the signal $u_{b}$ as a function of the time length $t$, in a log-log representation. We show the results corresponding to different partitions, all of them sharing the same power-law exponent 0.83 . From top to bottom: partition $\mathcal{P}_{100}, \mathcal{P}_{32}$, $\mathcal{P}_{8}, \mathcal{P}_{2}$. The bottom solid line is the analytical prescription for the power-law exponent 0.83 .

cess. We shall use this model to reproduce the Fourier spectrum, the DE and CIC properties of the experimental data $u_{a}$ and $u_{b}$.

Actually, it should be emphasized that our purpose is not to buildup a simplified model that mimics the physics underlying the experimental data or even their exact time variation (in terms of instantaneous frequencies), but just to propose a model that produces time series having the same kind of complexity as the fluid dynamical data.

We shall give details only on the choices made to fulfill the conditions related $u_{b}$. The prescriptions adopted to create the model related to the signal $u_{a}$ are similar and, for the sake of simplicity, shall not be illustrated. First of all, we define the following normalized waiting time distribution:

$$
\Psi(\tau)=\frac{(\mu-1) T^{\mu-1}}{(T+\tau)^{\mu}} .
$$

In the case $\mu>2$, the first moment of this distribution is $T /(\mu-2)$, thereby showing that the parameter $T$ keeps the mean value of this time distribution under control. We also define the set of frequency values $A_{\ell}=\left\{\omega_{1}, \ldots \omega_{\ell}\right\}$, where the values $\omega_{j}$ 's are determined on the basis of the Hilbert transform approach used to derive the results of Fig. 4. More precisely, these values can be considered to be a coarsegraining representation of the condition illustrated in Fig. 3. In the specific case of the signal $u_{b}$, we have fixed $\ell=10$ and the frequencies $\omega_{j}$ 's have been determined by a uniform partition of the frequency centered at $(94.47 \mathrm{~Hz})$ with a width that is twice the standard deviation $(0.93 \mathrm{~Hz})$.

We now define the artificial signal as follows. We select a time $\tau_{1}$ of the distribution of Eq. (10), we call this time interval laminar phase and we randomly select one of the frequencies of the set $A_{\ell}$, say $\omega\left(\tau_{1}\right)$. We assume that the signal, a sinusoidal function of time, keeps this frequency throughout the whole laminar region. At the end of this lami- 
nar region, we select another number $\tau_{2}$ from the distribution $\psi(\tau)$ and another frequency $\omega\left(\tau_{2}\right)$ from the set $A_{\ell}$, and so on. At any random drawing of the pair $\left\{\tau_{j}, \omega\left(\tau_{j}\right)\right\}$, we must also select a phase $\phi_{j}$. This latter choice is not arbitrary, but it is done in such a way as to ensure that the resulting signal is continuous. In conclusion, we get the following signal:

$$
\sigma_{\mu}(t)=\sin \left[\omega_{\mu}(t) t+\phi_{\mu}(t)\right]
$$

where the subscript $\mu$ indicates the dependence of this artificial signal on the random distribution $\Psi$ of Eq. (10), with a fixed value of $\mu$. This special way of sewing a laminar region to the next has been dictated by the need of reproducing the experimental spectrum of Fig. 2. Our numerical calculations allowed us to assess that a different choice, based on the abrupt frequency jump, with a fixed phase, and, consequently, with an abrupt jump of the signal $\sigma_{\mu}(t)$, yields a continuous Fourier spectrum, thereby dramatically departing from the behavior illustrated by Fig. 2 .

Actually, the inverse power-law distribution of waiting times given by Eq. (10) might have been produced by the Manneville map. Indeed, we recall that the Manneville map $f(x)=x+x^{z}(\bmod 1)$, with $x \in[0,1]$, is characterized by a parameter $z>1$, which controls the frequency of the jumps from the random portion of the map to the laminar part of the map. Although the laminar portion of the map, which must be very close to the origin, is not defined without some ambiguity [16], it is possible to derive a distribution with the same asymptotic properties as the distribution of Eq. (10) using the following procedure. We call the return to the laminar region a "random event." We may consider the waiting time between two consecutive random events as time of sojourn in a condition of order. The corresponding distribution of waiting times is an inverse power law with index $\mu$ $=z /(z-1)$ [16]. The condition $z=2$ is the border between the region where the waiting time distribution has a finite mean $(z<2)$ and the region where this mean waiting time is infinite $(z>2)$. This latter behavior has been named sporadic dynamics [8]. Actually, the region $\frac{3}{2} \leqslant z<2$ is characterized by a waiting time distributions with divergent second moment. For what concerns the KS entropy, it is positive in the region $1<z<2$, while the entropy vanishes when the parameter $z$ enters the region $z \geqslant 2$. Hence, the Manneville map is weakly chaotic only when the parameter $z$ lies in the region $z \geqslant 2$.

Moreover, in both cases of time series drawn from a Manneville map with driving parameter $z>2$ and of time series generated by distribution (10) with $\mu=z /(z-1)<2$, it has been proved that the information content of the time series grows as a power law $I_{Z}(n) \sim n^{\alpha}$, where $\alpha=1 /(z-1)$ $=\mu-1<1$. The proof is a rigorous theorem in the first case $[11,12]$ and an experimental evidence in the second case [24].

Now, it comes as a natural consequence to determine, in the proposed model (11), the parameter $\mu$ that reproduces the kind of complexity of the reconstructed signals. Indeed, working out that value of $\mu$ corresponds to disclose the complexity of the hidden driving process $\omega_{\mu}(t)$ : the latter de-

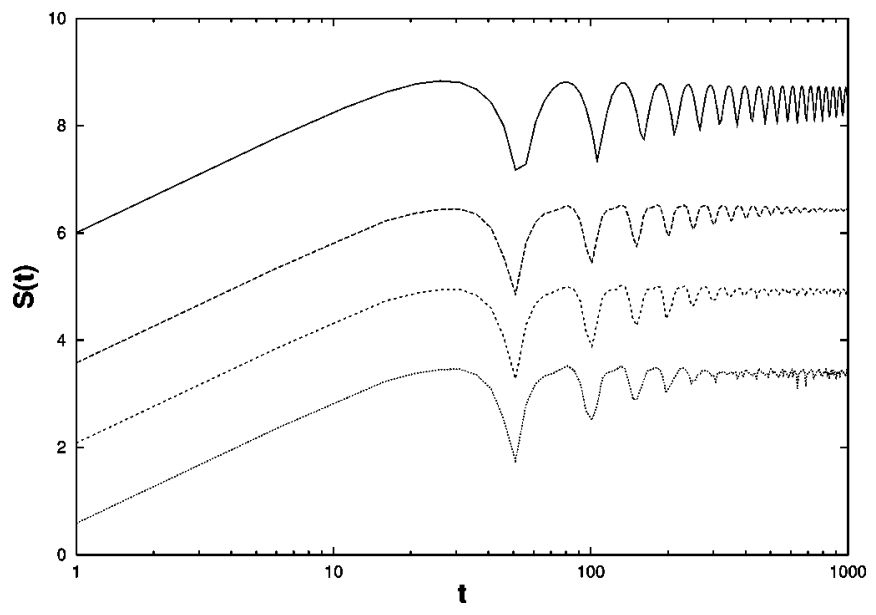

FIG. 6. The entropy $S(t)$ as a function of time. Results of the $\mathrm{DE}$ analysis on $u_{b}$ (top graph, solid line) and the simulated signals $\sigma_{\mu}(t)$ generated with different values of the control parameter. From top to bottom: $\mu=3.5, \mu=2.45, \mu=1.90$.

pends only on the frequency of the random events given by the function $\Psi(t)$ as shown in Ref. [24], [16].

As a first step, the model should reproduce the time evolution of the DE. As it is clearly illustrated in Fig. 6, the artificial sequences obtained from model (11) with three different values of $\mu(\mu=1.90, \mu=3.5, \mu=2.45)$ have the same behavior of $S(t)$ as the reconstructed signal $u_{b}$. The above choice of the values of $\mu$ is motivated by the different properties of the probability of the random events. Indeed, if $\mu>3$ both the mean value and the variance of $\Psi$ are finite, if $2<\mu \leqslant 3$ only the mean value is finite, whereas if $1<\mu$ $\leqslant 2$ both the mean value and the variance are infinite. Therefore, the DE is not sensitive to detect the exact value of $\mu$.

We note that, in spite of a lack of sensitivity to the complexity of the hidden driving process, Fig. 6 proves that there is a good agreement between the DE of the reconstructed signal $u_{b}$ and the $\mathrm{DE}$ of three simulated sequences generated with different driving parameter $\mu$. Therefore, Eq. (11), with the random choice of waiting times prescribed by Eq. (10), is a plausible model of the process under study. At the moment we do not have any analytical expression to relate the information content index $\alpha$ to the driving parameter $\mu$ of Eq. (11). Thus, it is impossible to determine theoretically the inverse power-law distribution and, thus, the complexity of the driving process. We must rest on a numerical treatment, based on applying the compression algorithm to artificial sequences with different values of $\mu$. For this purpose, we have turned the artificial time series generated by Eq. (11) into several symbolic sequences by means of a uniform partition $\mathcal{P}_{2}$. For all the values of $\mu$ adopted in the numerical simulations, the resulting information content of associated symbolic series turned out to be Eq. (9), with $\alpha<1$, which is equivalent to the sporadic randomness of Gaspard and Wang [8]. The dependence of $\alpha$ on $\mu$ is pictured in Fig. 7 and turned out to be essentially monotonic. The numerical function $\alpha(\mu)$ crosses the horizontal strip $0.80 \leqslant \alpha \leqslant 0.83$ at $\mu$ $=2 \pm 0.1$. Thus, the complexity of the driving stochastic process is measured, with a good approximation, by Eq. (11) with $\mu=2$. 


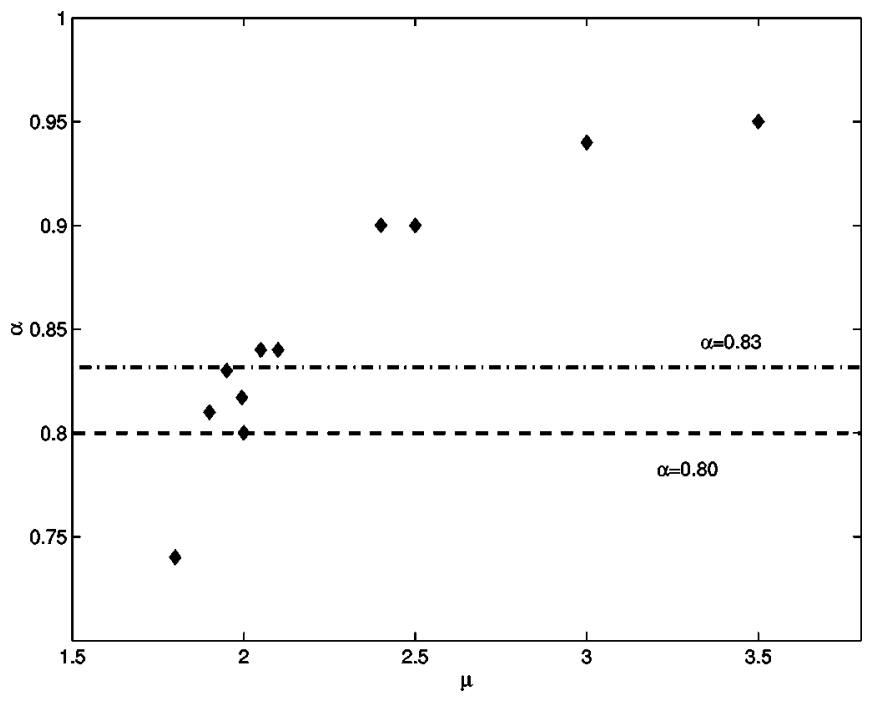

FIG. 7. The index $\alpha$ denoting the complexity of the signal $\sigma_{\mu}(t)$ of Eq. (11) as a function of the parameter $\mu$, denoting the complexity of the hidden driving process.

\section{CONCLUDING REMARKS}

The interest of this paper rests on the finding that the fluid dynamical process of the roll-up and pairing of vortices in a jet flow can be characterized as a weakly chaotic phenomenon. Indeed, the experimental data show unambiguously a vanishing $\mathrm{KS}$ entropy in spite of a nonregular evolution of the frequencies. Moreover, a finer indicator of the degree of complexity of the time series as the computable information content shows a power-law growth of the information with exponent $\alpha$ between 0.80 and 0.83 . In order to shed light on the complexity of the fluid dynamical processes, we proposed an artificial model where the hidden process is ruled by a power-law distribution of random events. The main properties (power spectrum, DE and CIC time evolution) of the experimental data are satisfactorily reproduced by the artificial signal of Eq. (11), obtained according to the procedure described in Sec. V. This important aspect is illustrated by Figs. 6 and 7. We devote these concluding remarks to further comments on the latter figure and we stress that two main results emerge from its analysis.
The first result is that the sewing process adopted to generate the artificial sequence yields a sort of time dilution of randomness. The driving signal $\omega_{\mu}(t)$ is characterized for $\mu>2$ by a nonvanishing Lyapunov coefficient and, consequently, by an information content growing linearly in time. The time increase of the information content becomes sublinear only in the region $\mu<2$. We see from Fig. 7 that the information content of the signal $\sigma_{\mu}(t)$ increases sublinearly in time throughout the whole considered range of $\mu$ values and remains probably sublinear well beyond $\mu=3$, which is the border between Lévy and ordinary Gaussian diffusion in the anomalous diffusion framework. According to the definition of weak chaos adopted in this paper, we conclude that the signal $\sigma_{\mu}$ is an expression of weak chaos regardless of the degree of hidden complexity. At the moment we do not have any theory expressing $\alpha$ as a function of $\mu$. However, the numerical results of Fig. 7 indicate that $\alpha(\mu)$ is a monotonic function of $\mu$ with $\alpha<1$. The second result has to do with the detection of the degree of complexity of the hidden driving signal $\omega_{\mu}(t)$. The uncertainty on the complexity of the signal $u$ is measured by the width of the strip between the straight line $\alpha=0.80$ and the straight line $\alpha=0.83$. Thus, the uncertainty on the complexity of the hidden signal may be suggested by observing the crossing between the curve $\alpha(\mu)$ and the strip. We see from Fig. 2 that the crossing occurs in a sharp interval of $\mu$ values around $\mu=2$. More precisely, the complexity of the driving signal is measured by $1.9<\mu$ $<2$.1. This leads us to conclude that the driving signal is ruled by a distribution of random events that lies at the border between nonstationary condition $(\mu<2)$ and infinite variance condition $(2<\mu<3)$. There are other complex processes lying very close to this border (see, for instance, the solar flares of Ref. [37] and the earthquakes in California [38]). It would be interesting to assess why the border between the stationary and the nonstationary states is the basin of attraction of some complex systems.

\section{ACKNOWLEDGMENT}

P.G. gratefully acknowledges financial support from ARO, through Grant No. DAAD19-02-0037.
[1] A. N. Kolmogorov, C. R. (Dokl.) Acad. Sci. URSS 119, 861 (1958); Ya. G. Sinai, ibid. 124, 768 (1959).

[2] G. M. Zaslavsky, R. Z. Sagdeev, D. A. Usikov, and A. A. Chernikov, Weak Chaos and Quasi-Regular Patterns (Cambridge University Press, Cambridge, England, 1991).

[3] X.-J. Wang, in Complexity, Entropy, and the Physics of Information, edited by W. H. Zurek, SFI studies in the Scences of Complexity Vol. VII (Addison-Wesley, Redwood City, CA, 1990), p. 319.

[4] J. P. Crutchfield and K. Young, in Complexity, Entropy, and the Physics of Information (Ref. [3]), p. 223.

[5] P. Bak, C. Tang, and K. Wiesenfeld, Phys. Rev. A 38, 364 (1988).
[6] G. M. Zaslavsky and M. Edelman, Chaos 11, 295 (2001).

[7] P. Manneville, J. Phys. (Paris) 41, 1235 (1980).

[8] P. Gaspard and X. J. Wang, Proc. Natl. Acad. Sci. U.S.A. 85, 4591 (1988).

[9] F. Argenti, V. Benci, P. Cerrai, A. Cordelli, S. Galatolo, and G. Menconi, Chaos, Solitons Fractals 13, 461 (2002).

[10] V. Benci, C. Bonanno, S. Galatolo, G. Menconi, and M. Virgilio, Discrete Contin. Dyn. Syst., Ser. B (to be published).

[11] C. Bonanno, http://arXiv.org/abs/math.DS/0107195

[12] S. Galatolo, Nonlinearity (to be published).

[13] C. Bonanno and G. Menconi, Discrete Contin. Dyn. Syst., Ser. B 2, 415 (2002).

[14] S. Montangero, L. Fronzoni, and P. Grigolini, Phys. Lett. A 
285, 81 (2001).

[15] G. Buresti and G. Lombardi, to appear in Proceedings of the Seventh National Congress on Wind Engineering.

[16] G. Aquino, P. Grigolini, and N. Scafetta, Chaos, Solitons Fractals 12, 2023 (2001).

[17] T. Geisel, J. Nierwetberg, and A. Zacherl, Phys. Rev. Lett. 54, 616 (1985).

[18] M. F. Shlesinger and J. Klafter, Phys. Rev. Lett. 54, 2552 (1985).

[19] M. F. Shlesinger, B. J. West, and J. Klafter, Phys. Rev. Lett. 58, 1100 (1987).

[20] M. Buiatti, P. Grigolini, and A. Montagnini, Phys. Rev. Lett. 82, 3383 (1999).

[21] M. Ignaccolo, P. Grigolini, and A. Rosa, Phys. Rev. E 64, 026210 (2001).

[22] M. Shlesinger, G. M. Zaslavsky, and J. Klafter, Nature (London) 363, 31 (1993).

[23] N. Scafetta, P. Hamilton, and P. Grigolini, Fractals 9, 193 (2001).

[24] P. Allegrini, V. Benci, P. Grigolini, P. Hamilton, M. Ignaccolo, G. Menconi, L. Palatella, G. Raffaelli, N. Scafetta, M. Virgilio, and Y. Yang, Chaos, Solitons Fractals 15, 17 (2003).

[25] G. Buresti, P. Petagna, and A. Talamelli, Exp. Therm. Fluid Sci. 17, 18 (1998).

[26] R. Carmona, W. L. Hwang, and B. Torresani, Practical Time-
Frequency Analysis (Academic Press, San Diego, CA, 1998).

[27] P. Grigolini, L. Palatella, and G. Raffaelli, Fractals 9, 439 (2001).

[28] W. Feller, An Introduction to Probability Theory and Its Applicaton (Wiley, New York, 1971).

[29] G. J. Chaitin, Information, Randomness and Incompleteness, Papers on Algorithmic Information Theory (World Scientific, Singapore, 1987).

[30] A. A. Brudno, Trans. Mosc. Math. Soc. 2, 127 (1983).

[31] S. Galatolo, Discrete Contin. Dyn. Syst. 7, 477 (2001).

[32] S. Galatolo, Nonlinearity 13, 1531 (2000).

[33] T. H. Solomon, E. R. Weeks, and H. L. Swinney, Phys. Rev. Lett. 71, 3975 (1993).

[34] L. Bonci, R. Roncaglia, B. J. West, and P. Grigolini, Phys. Rev. Lett. 67, 2593 (1991)

[35] L. Fronzoni, P. Grigolini, and S. Montangero, Chaos, Solitons Fractals 11, 2361 (2000).

[36] M. T. Rosenstein, J. J. Collins, and C. J. De Luca, Physica D 65, 117 (1993).

[37] P. Grigolini, D. Leddon, and N. Scafetta, Phys. Rev. E 65, 046203 (2002).

[38] S. Mega, P. Allegrini, P. Grigolini, V. Latora, L. Palatella, A. Rapisarda, and S. Vinciguerra, Phys. Rev. Lett. 90, 188501 (2003). 